\title{
Transport through a quantum dot analyzed by electron counting
}

Christian Fricke, Frank Hohls, Nandhavel Sethubalasubramanian, Lukas Fricke, and Rolf J. Haug

Citation: AIP Conference Proceedings 1399, 305 (2011);

View online: https://doi.org/10.1063/1.3666375

View Table of Contents: http://aip.scitation.org/toc/apc/1399/1

Published by the American Institute of Physics 


\title{
Transport through a quantum dot analyzed by electron counting
}

\author{
Christian Fricke, Frank Hohls,2 Nandhavel Sethubalasubramanian, Lukas Fricke and \\ Rolf J. Haug
}

Institut für Festkörperphysik, Leibniz Universität Hannover, D-30167 Hannover, Germany

\begin{abstract}
We present measurements of single electron tunneling through a quantum dot (QD) using a quantum point contact as charge detector. The counting statistics for varying symmetry of the QD system is examined from a very large statistical basis. We extract high order cumulants describing the distribution and observe prominent oscillations when varying the symmetry. We compare this behavior to the observed oscillation in time dependence and show that the variation in both system variables lead to the same kind of oscillating response.
\end{abstract}

Keywords: Quantum Dots, Full Counting Statistics, Cumulants

PACS: $73.23 . \mathrm{Hk}, 73.40 . \mathrm{Gk}, 73.63 . \mathrm{Kv}, 73.63 . \mathrm{Rt}, 02.50 .-\mathrm{r}$

Current fluctuations in mesoscopic systems allow to obtain information on transport that can not be observed in the average current [1]. The shot noise has been measured as a first step beyond the mean to examine correlations in the electron transport through semiconducting quantum dot systems [3, 4], but the extraction of higher moments from the current fluctuations is a challenging task [5] and has not yet been achieved for transport through quantum dots. In the context of full counting statistics (FCS) higher moments are directly accessible [7, 6]. With the use of a quantum point contact (QPC) as a charge detector with fast time response, FCS became experimentally feasible in quantum dot physics $[8,9,10,11]$. A significantly improved experimental technique has made the extraction of very high-order cumulants possible, revealing an oscillating behavior as function of integration time that strongly increases with the order of the moment $[12,13]$. The theoretical treatment has shown that these oscillations are a universally expected phenomenon for most physical systems and that in quantum dots they should show up prominently as function of the barrier asymmetry[16, 12]. Recently this prediction was also confirmed experimentally [17].

A three dimensional AFM image of our device is presented in Fig. 1. The elevated lines depict the insulating oxide barriers written by the AFM. The QPC (lower area) is separated from the QD structure (upper area) by an insulating line. The QPC can be electrically tuned using the in-plane gate GQ. The current through the QPC is amplified by a current amplifier and detected in a time-

\footnotetext{
1 fricke@nano.uni-hannover.de

2 present adress: Physikalisch-Technische Bundesanstalt, D-38116 Braunschweig, Germany
}
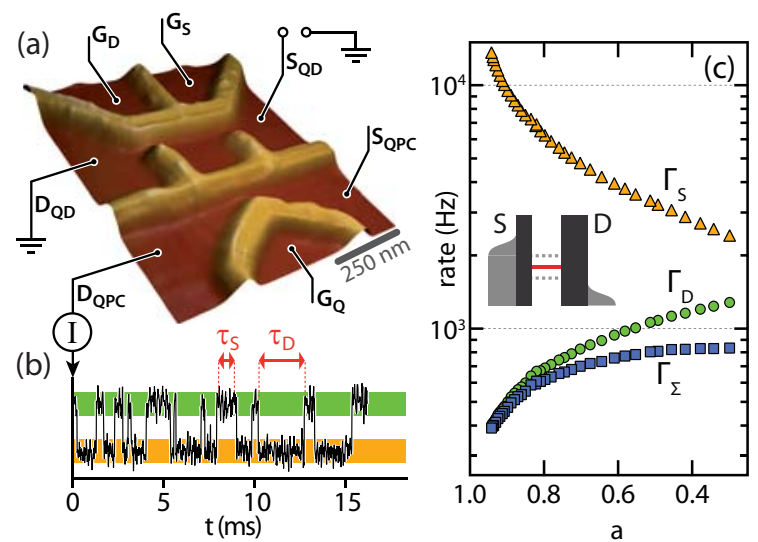

FIGURE 1. (a) Atomic force microscope topography of quantum dot (QD) and quantum point contact (QPC) Single electrons enter the QD from the source $\left(S_{Q D}\right)$ and leave via the drain $\left(D_{Q D}\right)$. (b) The current running through the QPC switches back and forth between a high and a low level as an electron enters and leaves the QD. This allows us to count the number of electrons that have passed through the QD in real-time. A typical time-trace of the QPC current is shown. The tunneling rates can be extracted from the waiting times $\tau_{S}$ and $\tau_{D}$. (c) Measured tunneling rates $\Gamma_{S}$ (source), $\Gamma_{D}$ (drain) and the electron transport rate $\Gamma_{\Sigma}$ with respect to the asymmetry parameter $a$. The asymmetry parameter is modified by changing the quantum dot gate configuration.

resolved manner. The bandwidth of the charge detection is only limited by the current amplifier bandwidth and slightly exceeds $100 \mathrm{kHz}$. The QPC bias is chosen sufficiently small to avoid back-action on the QD. The QD is coupled to source and drain electrodes via two tunneling barriers which can be separately controlled with the 

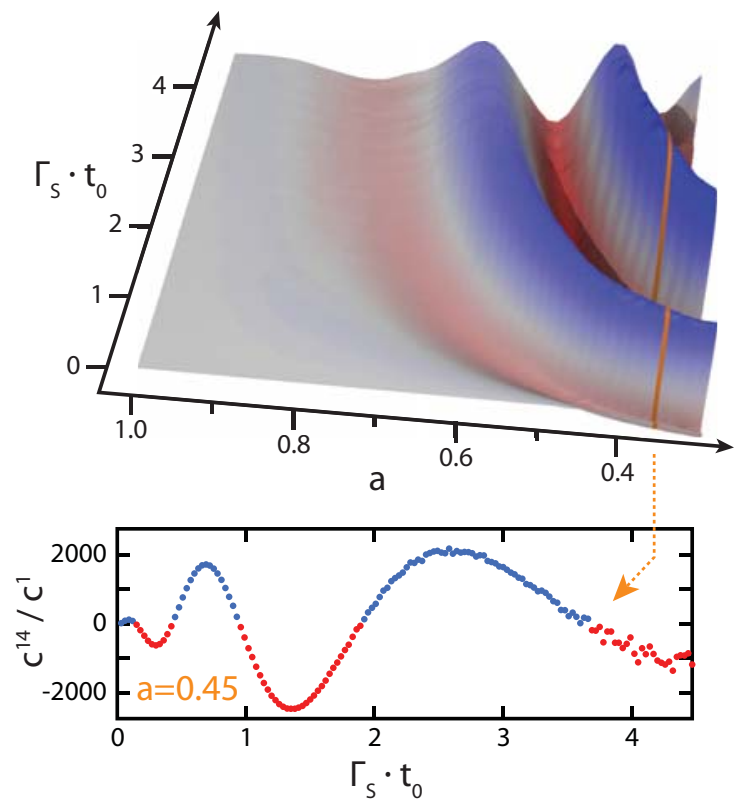

FIGURE 2. Symmetry and time dependence of the 14th cumulant. Strong oscillations appear displaying the interplay of different system parameters. In the lower plot a cut at $a=0.45$ is shown.

in-plane gates GS and GD. These gates are also used to tune the number of electrons on the QD. To analyze only unidirectional transport, the system is tuned to a situation where the transport state of the dot is sufficiently far from the source and drain Fermi energies, thus allowing no back-tunneling from the dot to the source contact or from drain to the dot (compare inset in Fig. 1b). From the real-time charge signal we extract the full counting statistics of the electrons participating in transport through the QD. In theory it is stated that high-order cumulants of the FCS show oscillations for nearly any system when changing a relevant parameter, e.g. the symmetry of the tunneling rates in a quantum dot system [12]. For our experimental study of this predicted symmetry dependence of high-order cumulants we varied the asymmetry parameter $a=\left(\Gamma_{S}-\Gamma_{D}\right) /\left(\Gamma_{S}+\Gamma_{D}\right)$ and measured for each $a$ about 1 million tunneling events.

We can thereby determine the dependence on the time (displayed here as the dimensionless length of the counting interval $\Gamma_{S} \cdot t_{0}$ ) for each value of the asymmetry $a$, thereby mapping out the cumulants as function of both parameters. The result obtained for the normalized 14th cumulant $c^{14} / c^{1}$ is shown in Fig. 2. The figure nicely reveals the similarity in the dependencies on asymmetry and on dimensionless time - the general features are nearly symmetric with respect to the diagonal. This correspondence between the different parameters demon- strates that the occurrence and the strength of these oscillations are of universal nature [12]. It is also interesting to examine the behavior at long times or towards the case of symmetric rates, $a=0$. In both cases the dependence on the diametrical parameter becomes weak and the cumulants are governed only by one parameter. This transitions again reveal the similarities in the dependence on these different parameters and support the underlying universal nature of the oscillatory behavior.

We thank C. Flindt for many fruitful discussions. The work was supported by the Federal Ministry of Education and Research of Germany via nanoQUIT and the German Excellence Initiative via QUEST.

\section{REFERENCES}

1. Y. M. Blanter, M. Buttiker, Physics Reports 336 (1-2) (2000) 1-166.

2. H. Birk, M. J. M. de Jong, and C. Schöneberger, Phys. Rev. Lett., 75(8):1610, 1995

3. A. Nauen, I. Hapke-Wurst, F. Hohls, U. Zeitler, R. J. Haug, and K. Pierz, Phys. Rev. B, 66(16):161303, 2002.

4. A. Nauen, F. Hohls, N. Maire, K. Pierz, and R. J. Haug, Phys. Rev. B, 70(3):0333305, 2004.

5. B. Reulet, J. Senzier, and D. E. Prober, Phys. Rev. Lett. 91:196601, 2003.

6. D. A. Bagrets and Yu. V. Nazarov, Phys. Rev. B, 67(8):085316, 2003.

7. L. S. Levitov, H. Lee, and G. B. Lesovik. J. Math. Phys., 37(10):4845, 1996.

8. S. Gustavsson, R. Leturcq, B. Simovic, R. Schleser, T. Ihn, P. Studerus, K. Ensslin, D. C. Driscoll, and A. C. Gossard. Phys. Rev. Lett., 96(7):076605, 2006.

9. T. Fujisawa, T. Hayashi, R. Tomita, and Y. Hirayama, Science, 312:1634, 2006.

10. C. Fricke, F. Hohls, W. Wegscheider, and R. J. Haug, Phys. Rev. B, 76(15):155307, 2007.

11. S. Gustavsson, R. Leturcq, T. Ihn, K. Ensslin, M. Reinwald, and W. Wegscheider, Phys. Rev. B, 75(7):075314, 2007.

12. C. Flindt, C. Fricke, F. Hohls, T. Novotný, K. Netocný, T. Brandes, and R. J. Haug, Proc. Natl. Acad. Sci. U.S.A., 106(25): 10116, 2009.

13. C. Fricke, F. Hohls, C. Flindt, and R. J. Haug, Physica E, 42(4):848, 2010.

14. I. M. Dremin, and R. C. Hwa. Phys. Rev. D, 49(11):5805, 1994.

15. V.V. Dodonov, I.M. Dremin, P.G. Polynkin, and V.I. Man'ko. Physics Letters A, 193(3):209, 1994.

16. C. Flindt, T. Novotný, A. Braggio, M. Sassetti, and A. Jauho. Phys. Rev. Lett., 100:150601, 2008.

17. C. Fricke, F. Hohls, N. Sethubalasubramanian, L. Fricke, R. J. Haug Appl. Phys. Lett., 96:202103, 2010.

18. R. Held, T. Vancura, T. Heinzel, K. Ensslin, M. Holland, and W. Wegscheider. Appl. Phys. Lett., 73(2):262, 1998.

19. U. F. Keyser, H. W. Schumacher, U. Zeitler, R. J. Haug, and K. Eberl. Appl. Phys. Lett., 76(4):457, 2000. 